\title{
High-throughput proteogenomics of Ruegeria pomeroyi: seeding a better genomic annotation for the whole marine Roseobacter clade
}

\author{
Joseph A Christie-Oleza, Guylaine Miotello and Jean Armengaud*
}

\begin{abstract}
Background: The structural and functional annotation of genomes is now heavily based on data obtained using automated pipeline systems. The key for an accurate structural annotation consists of blending similarities between closely related genomes with biochemical evidence of the genome interpretation. In this work we applied highthroughput proteogenomics to Ruegeria pomeroyi, a member of the Roseobacter clade, an abundant group of marine bacteria, as a seed for the annotation of the whole clade.

Results: A large dataset of peptides from R. pomeroyi was obtained after searching over 1.1 million MS/MS spectra against a six-frame translated genome database. We identified 2006 polypeptides, of which thirty-four were encoded by open reading frames (ORFs) that had not previously been annotated. From the pool of 'one-hitwonders', i.e. those ORFs specified by only one peptide detected by tandem mass spectrometry, we could confirm the probable existence of five additional new genes after proving that the corresponding RNAs were transcribed. We also identified the most-N-terminal peptide of 486 polypeptides, of which sixty-four had originally been wrongly annotated.

Conclusions: By extending these re-annotations to the other thirty-six Roseobacter isolates sequenced to date (twenty different genera), we propose the correction of the assigned start codons of 1082 homologous genes in the clade. In addition, we also report the presence of novel genes within operons encoding determinants of the important tricarboxylic acid cycle, a feature that seems to be characteristic of some Roseobacter genomes. The detection of their corresponding products in large amounts raises the question of their function. Their discoveries point to a possible theory for protein evolution that will rely on high expression of orphans in bacteria: their putative poor efficiency could be counterbalanced by a higher level of expression. Our proteogenomic analysis will increase the reliability of the future annotation of marine bacterial genomes.
\end{abstract}

\section{Background}

The first complete bacterial genome to be sequenced was that of Haemophilus influenza [1]. Seventeen years later, techniques for sequence determination and automated annotation tools have improved dramatically [2]. Genome sequences are now considered to be highly redundant and thus accurate when fully assembled. However, genome annotation is still far from being perfect, either in terms of structure (precise location of gene starts, regulatory sequences, etc.) or in terms of functional assignments $[3,4]$. An in-silico genome

\footnotetext{
* Correspondence: jean.armengaud@cea.fr

CEA, DSV, IBEB, Lab Biochim System Perturb, Bagnols-sur-Cèze, F-30207, France
} prediction in some prokaryotic genomes [5]. The genomes of almost 1600 living cellular organisms from the three domains of life have been sequenced and annotated to date: 1460 bacteria, 105 archaea, and forty eukarya (2011/05/21 update). The annotation of subsequent thousands of genomes expected to be released within the coming months (the annotation of 4906 microbial genomes is currently in progress) will rely, in almost all cases, on automated annotation pipelines and will be deposited as such in repository databases with no manual verification [6].

New strategies have been proposed to better annotate genomes with the integration of experimental data 
collected at the transcriptome or proteome levels (for a review, see: [7]). The expressed genome can give a reliable refinement of genome annotation and can be further extended to other related genomes by comparative genomics. In this way, massive transcriptome sequencing (RNA-seq) has been carried out for Caenorhabditis elegans [8] and Vitis vinifera [9], producing a large list of novel, transcribed sequences and alternative splicing information. However, many RNAs are noncoding and, therefore, coding RNAs that exhibit low similarities with other sequences should be further confirmed. Hence, a more direct analysis of proteins is recommended. Recent improvements in mass spectrometry have allowed high-throughput protein analysis by shotgun nanoLC-MS/MS, which can generate useful information on thousands of proteins $[10,11]$. The integration of proteomic data into a nucleotide database translated in the six reading frames, in order to improve genome annotation, was first proposed by Yates and coworkers [12] and has subsequently been applied at a large genomic scale by many research groups [3]. The resulting information is used to identify novel genes that were missed in the first annotation and to correct annotation mistakes [7]. The mapping of mass spectrometrycertified peptides onto the nucleotide sequence has been applied at the primary annotation phase for at least three microorganisms: Mycoplasma mobile [13], Deinococcus deserti [14], and Thermococcus gammatolerans [15]. Integrating both transcriptomic and proteomic complementary approaches has already been carried out for Pristionchus pacificus [16] and Geobacter sulfurreducens [17]. The main drawback of both approaches is that only a fraction of the transcriptome or the proteome can generally be observed under standard laboratory culture conditions for generalist lifestyle organisms, i.e. those with large genomes [18].

On the basis of evolutionary constraints, re-annotations obtained by proteogenomics for one organism can be extended to all orthologous genes present in all phylogenetically related species. This approach, first proposed by Gallien et al. [19] for the Mycobacterium genus, was tentatively called ortho-proteogenomics. A similar study has also been performed on Yersinia pestis KIM and extended, respectively, to the twenty-one closest Yersinia species sequenced [20]. The integration of proteogenomic studies carried out on closely related species addresses several notorious problems encountered with mass spectrometry approaches. Such is the case of the 'one-hit-wonders', proteins detected with only one MS/MS identified peptide which need to be evaluated with caution.

The Roseobacter clade is a group of bacteria that can represent up to $20 \%$ of bacterioplankton communities in coastal environments and more than $10 \%$ in the open ocean [21]. The first Roseobacter genome to be sequenced was that of Ruegeria pomeroyi DSS-3. This genome consists of a $4.11 \mathrm{Mb}$ chromosome and a 0.49 $\mathrm{Mb}$ megaplasmid, named pDSS-3 [22]. A high number of Roseobacter isolates have since been sequenced and many more are in progress in order to explore this important clade and to understand how ocean ecosystems function.

In the present study, we propose a re-annotation of the $R$. pomeroyi genome using experimental data obtained from a large proteomic survey recorded for this micro-organism. This ortho-proteogenomic study is the first to propose the extension of proteogenomic corrections to a large bacterial clade. Moreover, we have detected highly abundant orphan proteins and discuss their significance.

\section{Results}

Proteogenomic strategy for the analysis of $R$. pomeroyi

An extensive analysis of the proteome of $R$. pomeroyi, cultivated in various conditions, resulted in a large dataset of MS/MS spectra $(1,117,372)$. To assign these spectra to peptide sequences, we created a database comprising all translated stop-to-stop amino acid sequences of more than forty residues, taking into account the six-frame translation of the genome of $R$. pomeroyi. Thus, this database comprises all the coding domain sequences (CDS) of the bacterium mixed with a large number of aberrant polypeptide sequences. All of the MS/MS spectra were searched against this database using the Mascot engine, resulting in the identification of a restricted set of 4425 probable ORFs. A second search against this restricted database led to the assignment of 594 902 spectra corresponding to 22805 nonredundant tryptic peptides (Additional file 1). These peptides validated the presence in the cells of 2006 polypeptides detected with at least two peptides (Additional file 2). When comparing these mass spectrometry-certified ORFs with the 4252 previously annotated CDSs [22], we found that thirty-four ORFs were missing. Table 1 lists the corresponding proteins with their probable start and stop positions, as well as their putative function. Remarkably, most of these are unknown. We also took into consideration those novel ORFs detected with only one highly confident peptide ( $p$-value under $3.2 \mathrm{e}-5)$ and showing no overlap with other proteomicdetected genes. Applying these criteria resulted in the selection of seven targets. To give higher confidence to the validation of these 'one-hit-wonders', we checked whether these genes were transcribed. We extracted total RNA from bacteria grown in the physiological conditions in which each polypeptide had been most abundantly detected during the proteomic survey. We performed RT-PCR amplification for five of these targets 
Table 1 List of novel genes found in the genome of R.pomeroyi detected by proteogenomics.

\begin{tabular}{|c|c|c|c|c|c|c|c|}
\hline & Target $^{a}$ & $\begin{array}{l}\text { Plausible CDS } \\
\text { start }\end{array}$ & Stop & $\begin{array}{l}\text { Length } \\
\text { (aa) }\end{array}$ & $\begin{array}{l}\text { Peptides } \\
\text { assigned }\end{array}$ & $\begin{array}{l}\text { CDS Proteomic } \\
\text { coverage }\end{array}$ & $\begin{array}{l}\text { Function/Presence in other } \\
\text { Roseobacter stains }{ }^{b}\end{array}$ \\
\hline \multirow{26}{*}{$\begin{array}{l}\text { Non-annotated } \\
\text { CDS }\end{array}$} & SPOA_PG001 & 300016 & 300507 & 164 & 10 & $73 \%$ & Unknown/observed (9e-51) \\
\hline & SPO_PG002 & 3171305 & 3170874 & 144 & 9 & $69 \%$ & Unknown/unique \\
\hline & SPO_PG003 & 1412876 & 1413418 & 181 & 7 & $73 \%$ & Unknown/observed (5e-23) \\
\hline & SPOA_PG004 & 87032 & 87709 & 226 & 6 & $27 \%$ & Unknown/unique \\
\hline & SPO_PG005 & 358784 & 358125 & 220 & 6 & $45 \%$ & Esterase-lipase/observed (5e-45) \\
\hline & SPO_PG006 & 360911 & 360405 & 169 & 5 & $54 \%$ & Unknown/unique \\
\hline & SPO_PG007 & 1483195 & 1482533 & 221 & 5 & $48 \%$ & Unknown/unique \\
\hline & SPO_PG008 & 1431167 & 1431595 & 143 & 5 & $45 \%$ & Unknown/observed (3e-56) \\
\hline & SPO_PG009 & 501740 & 502171 & 144 & 5 & $42 \%$ & Unknown/unique \\
\hline & SPO_PG010 & 2353576 & 2353965 & 130 & 4 & $42 \%$ & Unknown/observed (1e-38) \\
\hline & SPO_PG011 & 1374589 & 1374299 & 97 & 3 & $61 \%$ & Unknown/conserved (1e-43) \\
\hline & SPO_PG012 & 3703461 & 3702955 & 169 & 3 & $22 \%$ & Unknown/unique \\
\hline & SPO_PG013 & 649156 & 649749 & 198 & 3 & $23 \%$ & Unknown/unique \\
\hline & SPO_PG014 & 2482691 & 2482317 & 125 & 3 & $20 \%$ & Unknown/unique \\
\hline & SPO_PG015 & 3657397 & 3656924 & 158 & 3 & $19 \%$ & Unknown/observed (6e-50) \\
\hline & SPO_PG016 & 373055 & 373333 & 93 & 2 & $41 \%$ & Unknown/unique \\
\hline & SPO_PG017 & 1092236 & 1092592 & 119 & 2 & $34 \%$ & Unknown/unique \\
\hline & SPO_PG018 & 495167 & 495529 & 121 & 2 & $22 \%$ & Unknown/observed (4e-44) \\
\hline & SPO_PG019 & 1418666 & 1419187 & 174 & 2 & $10 \%$ & Signal transduction/conserved (1e-69) \\
\hline & SPO_PG020 & 2807747 & 2807223 & 175 & 2 & $19 \%$ & Polyketide cyclase/unique \\
\hline & SPO_PG021 & 1289473 & 1289829 & 119 & 2 & $28 \%$ & Unknown/unique \\
\hline & SPO_PG022 & 1151078 & 1151632 & 185 & 2 & $18 \%$ & Unknown/unique \\
\hline & SPO_PG023 & 1400166 & 1399696 & 157 & 2 & $24 \%$ & Unknown/unique \\
\hline & SPO_PG024 & 2628409 & 2629668 & 420 & 2 & $9 \%$ & RNA helicase/conserved (1e-175) \\
\hline & SPO_PG025 & 1322016 & 1322357 & 114 & 1 & $7 \%$ & Transcriptional regulator/unique \\
\hline & SPO_PG026 & 3883013 & 3882531 & 161 & 1 & $7 \%$ & Unknown/unique \\
\hline \multirow[t]{9}{*}{ Wrong CDS } & SPO_PG027 & 501090 & 501710 & 207 & 21 & $77 \%$ & Unknown/unique \\
\hline & SPO_PG028 & 2429044 & 2427941 & 368 & 20 & $63 \%$ & Unknown/conserved (5e-92) \\
\hline & SPO_PG029 & 3124885 & 3123728 & 386 & 11 & $36 \%$ & Sporulation related/conserved (6e-92) \\
\hline & SPO_PG030 & 1738173 & 1736680 & 498 & 7 & $24 \%$ & Unknown/conserved (1e-175) \\
\hline & SPO_PG031 & 2905673 & 2906335 & 221 & 6 & $37 \%$ & Unknown/unique \\
\hline & SPO_PG032 & 3751605 & 3751147 & 153 & 6 & $42 \%$ & Unknown/conserved (5e-42) \\
\hline & SPO_PG033 & 2357076 & 2357507 & 144 & 2 & $18 \%$ & Excinuclease/observed (4e-35) \\
\hline & SPO_PG034 & 934724 & 935068 & 115 & 1 & $17 \%$ & Unknown/Observed (1e-27) \\
\hline & SPO_PG035 & 2751483 & 2750281 & 401 & 1 & $4 \%$ & Unknown/Conserved (1e-162) \\
\hline \multirow[t]{4}{*}{ Seq. error } & SPO_PG036 & 562052 & 560282 & 590 & 3 & $9 \%$ & ABC transporter/conserved $(0.0)$ \\
\hline & SPO_PG037 & 3188876 & 3188459 & 139 & 3 & $27 \%$ & Heat shock protein/observed (3e-55) \\
\hline & SPO_PG038 & 2152217 & 2151179 & 346 & 2 & $10 \%$ & Aminotransferase/conserved (1e-168) \\
\hline & SPO_PG039 & 3515528 & 3515111 & 139 & 1 & $17 \%$ & $\begin{array}{l}\text { Stress protein/unique (conserved in } \\
\text { Bacillus) }\end{array}$ \\
\hline
\end{tabular}

a Targets in bold represent those "one-hit-wonders" validated by RT-PCR.

${ }^{b}$ Observed indicates presence of a similar gene in less than 5 other Roseobacter strains whereas Conserved means presence in over 20 of the 36 strains searched. E-value for BLAST analysis with its nearest homologue is indicated in brackets.

(Panel A of Figure 1; Table 1). Supplementary Additional file 4 shows the 181 non-redundant peptides assigned to the thirty-nine novel CDSs to be subsequently considered. The culture conditions and proteome fraction in which each peptide was best detected is also indicated. Regarding these CDSs, we propose a novel nomenclature to indicate that their existence was experimentally verified by proteogenomics. It consists of the introduction of the two letters ' $\mathrm{PG}$ ', standing for ProteoGenomics, and a number beside the SPO nomenclature for annotated CDSs encoded on the bacterial chromosome and SPOA for those coded by the pDSS-3 
A

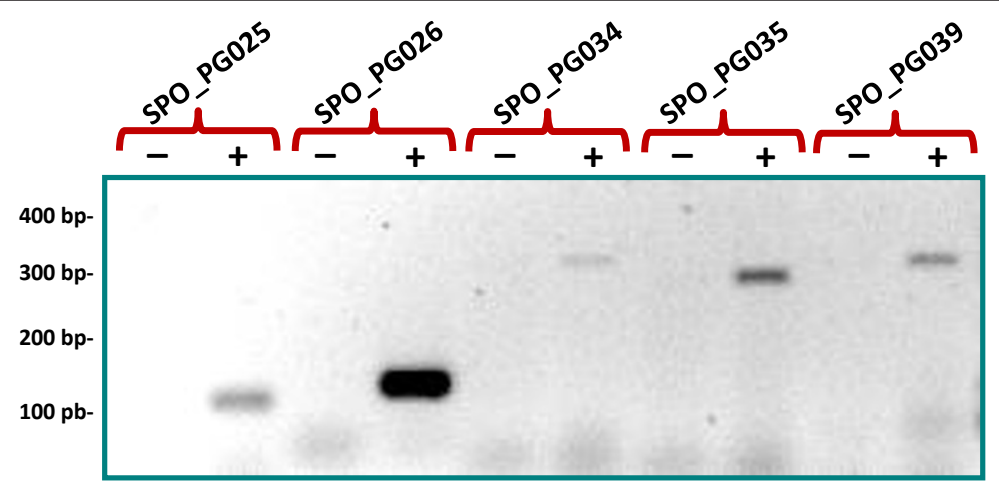

B

Proteomic evidence

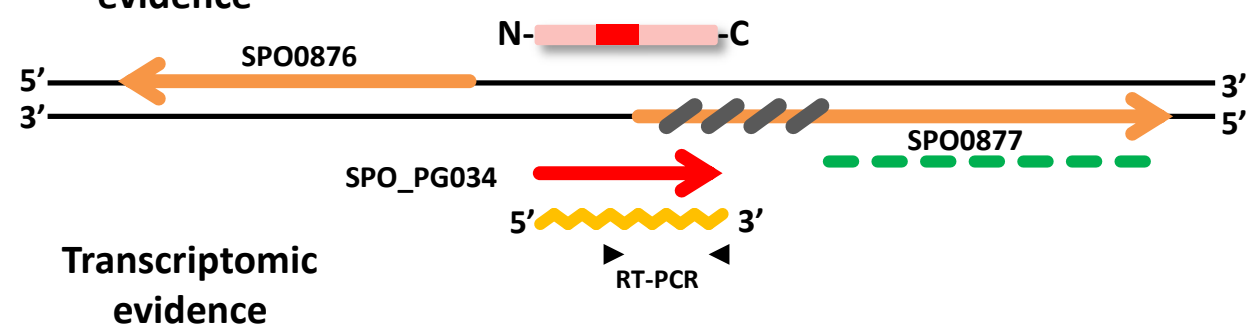

Figure 1 RT-PCR amplification for 'one-hit wonder' validation. A schematic view of the genomic region of target SPO_PG034 is shown as an example. The pink square represents the putative protein sequence highlighting in red the unique peptide detected by MS/MS. SPO0877 is shown with its conserved BLAST region (broken green line) and plausible non-coding area (grey crossed). In yellow is the mRNA produced from SPO_PG034 which was amplified with by RT-PCR using specific primers. The 3\% agarose gel stained with ethidium bromide shows the five "Onehit wonder" targets from which RT-PCR amplification was obtained (lane " + "). Lanes marked with "-" represent negative controls by testing PCR amplification on RNA extractions to ensure total DNA elimination.

megaplasmid (Table 1). We also identified ten ORFs with peptides located further upstream than their annotated N-termini (Additional file 5). This clearly indicated a wrongly identified translation start codon for the corresponding gene. We confirmed these corrections by Blast analysis. In addition, we listed in a second query the semi-tryptic peptides that could correspond to the most-N-terminal peptide, as detailed elsewhere [23].

\section{Novel CDSs found in, a priori, non-coding genomic regions}

As shown in Table 1, the SPO_PG001-026 CDSs were found to be encoded in genomic regions encompassing no previously annotated genes. Among them, sixteen have no known homologues in any other sequenced Roseobacter strains, while seven are homologous to proteins found in a limited number of Roseobacter strains. Generally, these genes encode small polypeptides (with a mean length of 166 amino acids) whereas the mean length of polypeptides encoded in the genome is greater than 320 amino acids. Curiously, the well conserved
RNA helicase, SPO_PG024, was not previously annotated, due to the report of a putative translational frameshift in the sequence [22]. We definitively discarded this possibility due to the detection of a peptide situated from amino acid position 432 to 454 in the stop-to-stop amino acid sequence, with the hypothetical frameshift in position 451 . The sequence between this position and the end of SPO_PG024 is badly conserved among the different Roseobacter members, being a possible hotspot for the accumulation of mutations and the reason why a putative frameshift was at first predicted.

\section{Novel CDSs indicating badly-annotated genes}

SPO_PG027-035 CDSs were found to overlap with previously annotated CDSs which had not been detected during the proteomic analysis. Moreover, the previously annotated CDSs did not exhibit any similarities with other bacterial proteins, as revealed by means of a PSIBLAST search. Seven of the new proposed genes whose products were detected by tandem mass spectrometry showed high similarities to proteins encoded in other 
related members of the Roseobacter clade (Table 1). Panel A of Figure 2 represents the chromosome region view of the target SPO_PG032. It shows how the tryptic peptides identified allow a better definition of each CDS on the genome, with no overlap between them. This figure also illustrates how the detection of the novel ORF, SPO_PG032, is in total discordance with the possible existence of SPO3540.
Remarkably, twenty-one distinct peptides cover $77 \%$ of the full SPO_PG027 polypeptide sequence. This protein was detected abundantly in the experimental conditions tested in the present study, with 752 spectral counts assigned to it (Additional file 4). Its sequence does not exhibit any similarities to other known proteins in any other sequenced organism. This specific gene, found in the $R$. pomeroyi genome, probably plays an important

A

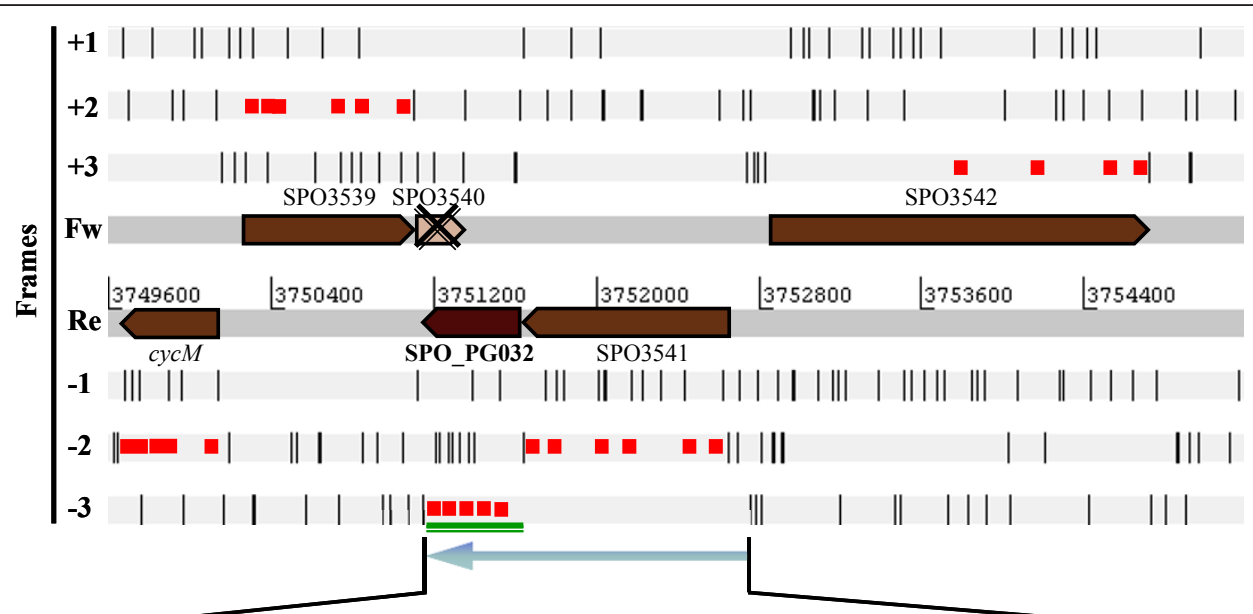

LVAICVNSAACRRH IGQFAIDLNRVSWQPFATGKPVNTKKERGRDENGRASHIRRLGPGPGGASARRWR GAEAHVGRSACALSVAVARQAAGRRRAAGTSGLGRPDPSAGDRGAGGRGRNADF PGPRRERRAALCTGH LGLGGRGAGPDRAGRVRRQIRAAAPRPATGAGLCRIATDHDPAQPARRRTGRHRQGGDRLACQP PVLRP LRRPQRDCPGGMATRVSGLWRTAFSAHRSGGDHADHPWRSGADGTLSGLARGHVFAACRICRTGRDAGG RRTPRGAGRGRRAGWCGHLSVQPALAVSRLADVRLRGRGPVAEPDHRPGRDRGCDLGQPQRHDAGLCRR APPPAACAQGGNCPFPVAELACGHAGLRGNSRKNHNRRGVMQFISKEDIEAPVAQVFAILSEFETFERS AMRR GVEVORVDASAPIGAGMAWDTRERLRGKPREMHVVLKOYDAPSLMR PASHSK GINGDVVIELLAL SPR RTRMSFVELKANTLSAFLFLOSLK ARKNMTRRFKLR IAEFAKEIESRCAR

B

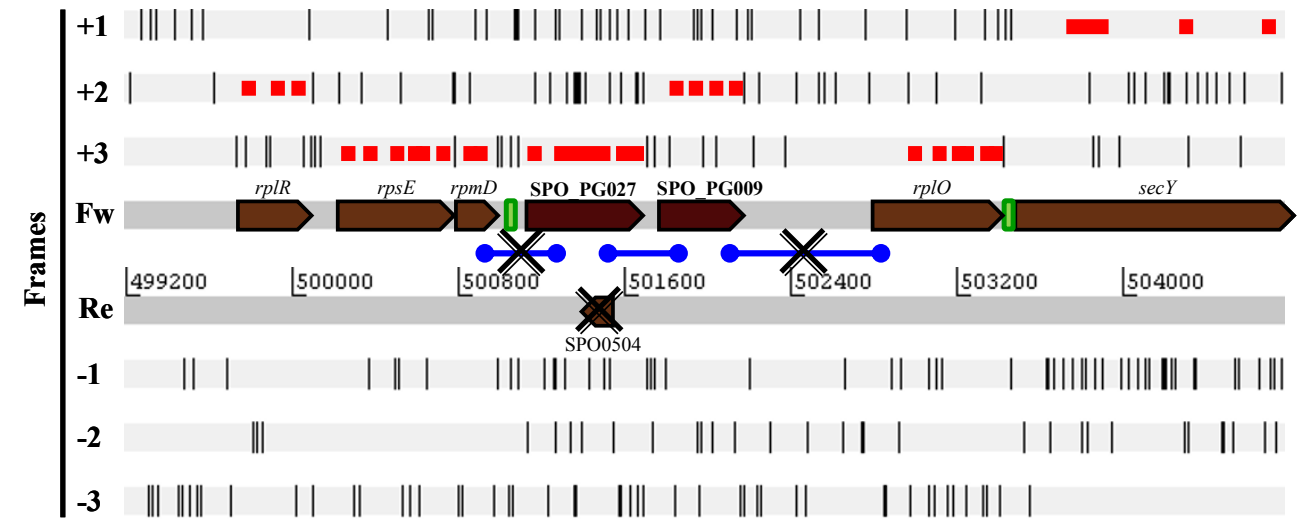

Figure 2 Chromosome region view of three novel genes detected by proteogenomics. Loci of targets SPO_PG032 (Panel A) and targets SPO_PG027 and SPO_PG009 (Panel B) are represented. The six reading frames are shown with all coded stop codons represented by black dashes. Coloured in red are the nucleic sequences specifying the peptides detected by MS/MS. Panel A: The green line represents the area of the stop-to-stop sequence that shows homology to other proteins by means of a PSI-BLAST. The amino acid sequence in bold black represents the plausible sequence of SPO_PG032. Highlighted in red squares are the peptides detected by MS/MS. Panel B: Blue lines represent RT-PCR amplification attempts. Green rectangles show identified transcriptional terminators. 
role in this bacterium as it was systematically detected in all of the biological samples analysed. Panel B of Figure 2 shows that this novel gene is found next to SPO_PG009, another re-annotated CDS, and both are located between two conserved ribosomal protein operons. Curiously, this intergenic region of $1.8 \mathrm{~kb}$ in $R$. pomeroyi presents a high variability among the closest sequenced Roseobacter members. This genomic area presented a variable size (0.1-1.5 kb) and sequence, with no inter-species similarity. The existence of the previously annotated SPO0504 gene is undoubtedly false. It was assigned to the other DNA strand and the corresponding short polypeptide product (forty-one residues) did not show any similar protein by PSI-BLAST. SPO_PG028 is also highly detected by MS/MS as 1395 spectra were assigned to this polypeptide (Additional file 4). In contrast to SPO_PG027, SPO_PG028 is highly conserved in other members of the Roseobacter clade. Curiously, SPO_PG028 was missed, favouring the annotated SPO2290 CDS, which gives no sequence homologue by means of PSI-BLAST.

Interestingly, the stop-to-stop ORF comprising SPO_PG031 overlapped the stop-to-stop ORF comprising an already annotated gene (SPO2724) detected during the proteomic survey. SPO2724 was validated with twenty non-redundant peptides (Additional file 2) but showed no similarities to other proteins by means of PSI-BLAST. The first validated peptide of SPO2724 was identified 901 nucleotides downstream of the initially annotated start codon. This indicates that this gene could be shorter, resulting in no overlap with SPO_PG031. A similar case is that of SPO_PG034 (onehit wonder example represented in Figure 1, Panel B). The C-terminal region of this newly detected gene overlaps the N-terminal region of the stop-to-stop SPO0877 ORF. A PSI-BLAST search with the annotated sequence of SPO0877 clearly indicates, by comparison with its closest homologues, that this conserved esterase lipase in $R$. pomeroyi should be 127 amino acids shorter. In such a case, the start codon of SPO0877 would be in position 935284 on the chromosome, being consistent with the existence of SPO_PG034.

\section{Detection of sequencing errors}

SPO_PG036-038 CDSs are highly conserved in other bacteria and all have putative assigned functions (Table 1). Like SPO_PG024, these genes were not considered at the primary stage of annotation (Moran 2004) as they all presented a putative frameshift in their sequence. For the targets SPO_PG036 and SPO_PG037, we detected two peptides located in one reading frame, as well as a third peptide that could belong to the same polypeptide but in another reading frame (Additional file 4). We double-checked the sequence of the nucleotide region where the plausible frameshift could occur and demonstrated a sequencing error in all four targets. In all cases we found an extra nucleotide in the sequence, which should be removed (an extra $\mathrm{G}$ at position 561 870, a $\mathrm{T}$ at position 3188 646, a $G$ at position 2151 427, and a $G$ at position 3 515150 , respectively, for each of the targets). These extra nucleotides led to changes in the reading-frame. The corrected protein sequences of these four targets are indicated in Additional file 4. They result in fulllength proteins with higher similarities with their respective homologues. SPO_PG039 is an interesting case revealed by the detection of a unique peptide. The corresponding polypeptide sequence does not share similarities with any protein present in other sequenced Roseobacter members, but has some similarity with a conserved stress response protein present in the genus Bacillus. As shown in Figure 3, the corresponding stop-to-stop ORF in the genome of $R$. pomeroyi overlapped the highly conserved annotated gene, SPO3316. The single peptide detected for this target, and the region exhibiting similarities to the stress response protein of Bacillus, both reside at the N-terminus of the ORF, in between the annotated genes, SPO3316 and SPO3317. When the extra G was removed, the reading-frame of SPO_PG039 was corrected, ending at a stop codon (position 3515 110) before overlapping SPO3316 (Figure 3).

\section{Polypeptide $\mathrm{N}$-terminus validation and correction}

To confirm or correct the annotation of translational start codons of the CDSs, we first searched for strictly tryptic peptides located upstream of those previously annotated in the stop-to-stop ORF sequence. In addition, we searched for semi-tryptic peptides to identify possible authentic N-terminal peptides.

The search for peptides located further upstream than the previously annotated start codons [22] led to the discovery of ten wrongly annotated CDSs. These are listed in Additional file 5. The ten resulting $\mathrm{N}$-terminal extensions did not overlap other genes. Moreover, higher similarities were found by PSI-BLAST with homologous genes annotated in other related species. Of note, one of these corrected start codons is that of the conserved $s u c B$ gene, SPO_0343, which is just downstream of sucA (SPO_0344), as shown in Figure 4. Another interesting case is that of SPO_1905, which shows high sequence similarities with its homologues found in the Roseobacter clade. However, the first residues were found to be very dissimilar in comparison with its closest homologues. We sequenced the locus and found a sequence error with an extra $G$ between positions 2029022 and 2029023 . This would be the reason why a shorter sequence was at first assigned to 


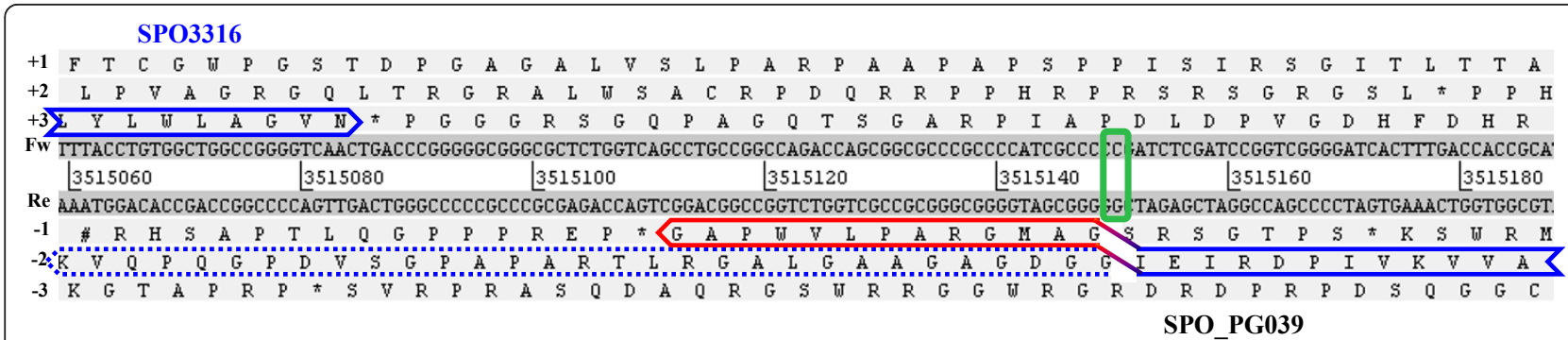

Figure 3 Locus of a sequencing error detected in the SPO_PG039 sequence. The reading frame of this novel detected gene shifts from frame -2 (highlighted in blue) to frame -1 (in red) due to an erroneously annotated extra nucleotide (highlighted in green).

this gene. The corrected N-terminus of the polypeptide sequence is now similar to its closest homologues.

The search for semi-tryptic peptides led to the discovery of the N-terminal peptides of 486 proteins (Additional file 6). All of these were confirmed by PSI-BLAST comparison with their counterparts in other species and by manual inspection: 422 peptides confirmed already well-annotated start codons, sixty-two peptides corresponded to genes with an erroneously identified start codon, and two were the $\mathrm{N}$-terminal peptides of the newly annotated genes, SPOA_PG001 and SPO_PG024, identified in this work (Table 1). These data reveal that almost $13 \%$ of the CDSs annotated at the primary stage [22] could have a wrongly identified start codon. As expected, the ratio of initiator codons is usual for bacteria: ATG (94\%), GTG (5\%) and TTG (1\%). Over 87\% $(54 / 62)$ of the wrongly annotated CDSs were shorter than previously annotated. This was as expected and is probably due to the GLIMMER annotation system used, which tends to privilege annotation producing the longest possible sequence.

\section{Seeding proteogenomic annotation for the whole Roseobacter clade}

An ortho-proteogenomic analysis was carried out in order to extend the genomic re-annotations proposed for $R$. pomeroyi to the thirty-six other sequenced Roseobacter members. For this, we performed a local tblastn to search for homologues of the thirty-nine new genes (Table 1) among the other thirty-six sequenced Roseobacter genomes. Table 2 compiles the eight homologous regions found that were not previously annotated as CDSs in their respective genomes. Six of these homologues belong to highly conserved genes and, as for $R$. pomeroyi, were simply missed during annotation. Interestingly, the novel identified targets, SPO_PG009 and SPO_PG020, showed a distant homologue in another Roseobacter strain (R. bacterium KLH11 and R. bacterium HTCC2083, respectively), meaning that these are no longer unique to $R$. pomeroyi.

The confidently detected $\mathrm{N}$-terminal sequences of the 486 CDSs listed in Additional file 6 were used to check whether some of their homologues found in the other

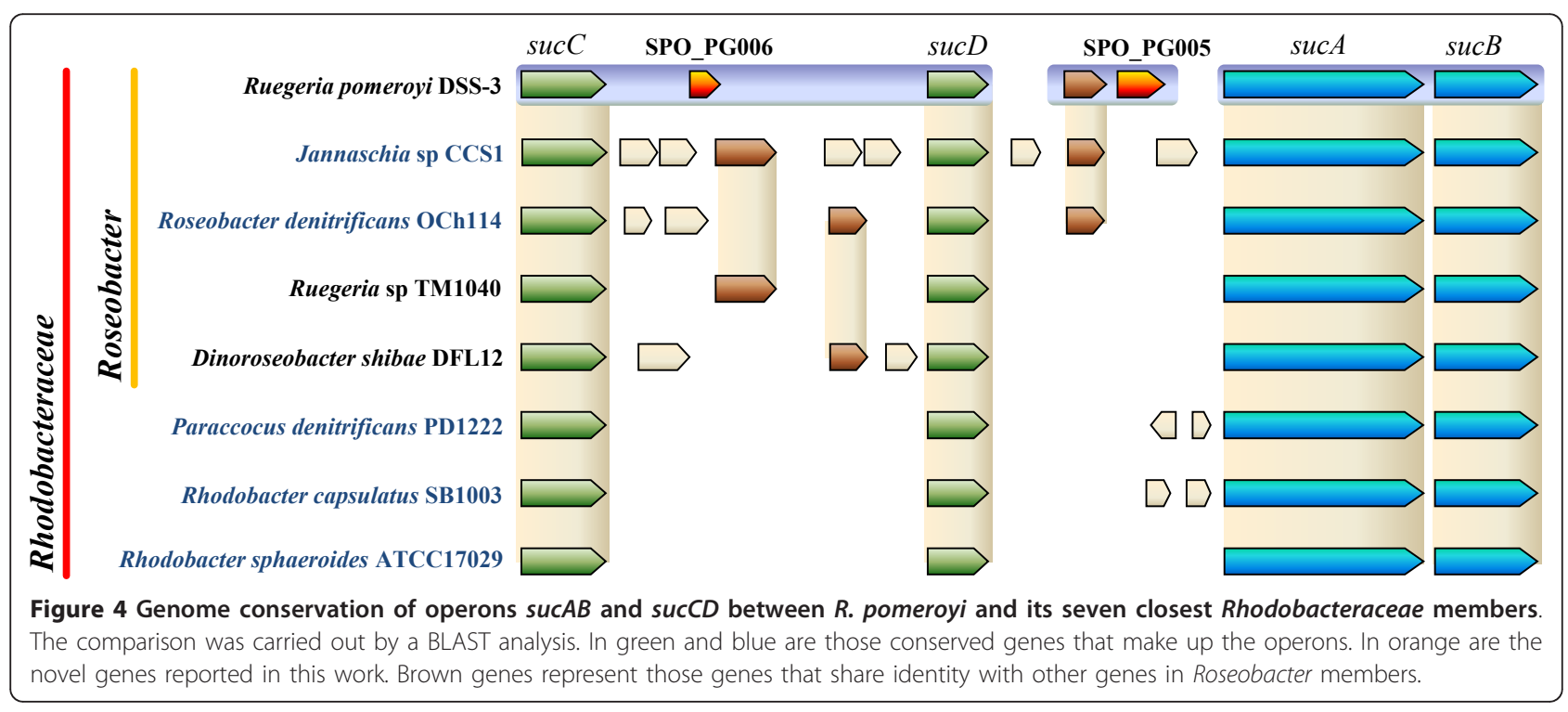


Table 2 List of novel genes detected after extending the data obtained in R.pomeroyi to 36 other Roseobacter members

\begin{tabular}{clcccc}
\hline Target & \multicolumn{1}{c}{ Roseobacter strain } & GenBank locus & 5' start & 3' stop & E-value \\
\hline SPO_PG009 & Rhodobacterales bacterium KLH11 & DS999531.1 & 1860966 & 1860490 & $4 \mathrm{e}-21$ \\
SPO_PG019 & Octadecabacter antarcticus 238 & DS990628.1 & 40225 & 39680 & $2 \mathrm{e}-27$ \\
& Octadecabacter antarcticus 307 & DS990575.1 & 845882 & 846427 & $5 \mathrm{e}-26$ \\
SPO_PG020 & Rhodobacterales bacterium HTCC2083 & DS995276.1 & 2703488 & 2704012 & $3 \mathrm{e}-26$ \\
SPO_PG024 & Phaeobacter sp. Y4I & DS995281.1 & 1272857 & 1271454 & $1 \mathrm{e}-158$ \\
SPO_PG029 & Roseobacter sp. MED193 & CH902583.1 & 691858 & 690500 & $3 \mathrm{e}-91$ \\
& Octadecabacter antarcticus 238 & DS990628.1 & 672254 & 670707 & $8 \mathrm{e}-33$ \\
SPO_PG032 & Rhodobacterales bacterium HTCC2083 & DS995276.1 & 1621388 & 1621861 & $4 \mathrm{e}-28$ \\
\hline
\end{tabular}

sequenced Roseobacter strains had been wrongly annotated. For this, a local tBLASTn analysis with the 486 protein sequences was performed for all the annotated CDSs of each of the thirty-six sequenced Roseobacters. A total of 9887 polypeptides sharing high similarities with these $R$. pomeroyi polypeptides were found and are listed in Additional file 7. The sequence and position of the first sixty amino acids of the alignment are also shown. In most cases, a consensus between the identified start codon of the $R$. pomeroyi protein and its respective homologue in other Roseobacter members was observed. Nevertheless, in 1082 cases the position of the most-N-terminal sequence annotated was not in agreement with the $\mathrm{N}$-terminus of the $R$. pomeroyi protein. For all of these, we propose the correction of the annotation after manual inspection. We checked whether the new gene starts corresponded to a canonical initiation codon (ATG, GTG, or TTG) and, in the case of a sequence extension at the $\mathrm{N}$-terminus, if there was evidence of sequence similarities with the $R$. pomeroyi polypeptide. The rate of erroneously identified start codons among the Roseobacter genomes was 11\% (1082 of 9887). Of note, this value may be an underestimate because i) we discarded many doubtful corrections during manual inspection, and ii) most of the BLAST results mainly matched highly conserved genes that are generally better annotated by comparative genomics. The rate decreased to $6.8 \%$ when considering only those curated genomes (i.e. indicated as "complete" in the Roseobase; D. shibae DFL12, R. denitrificans OCh114, Ruegeria sp. TM1040 and Jannaschia sp. CCS1).

\section{Defining operonic structures with re-annotated genes}

We found that some loci comprise several annotation errors. For example, Figure 2Panel B shows the locus where the two novel genes, SPO_PG09 and SPO_PG027, are encoded. As these two genes are equally oriented as the rpsE and $r p m D$ genes, both encoding conserved ribosomal proteins, we checked whether an operonic structure could be identified. We purified RNA from cells where the two proteins had been detected, and amplified specific cDNA fragments by RT-PCR (Figure 2, Panel B). We found that the two genes are co-transcribed, as we obtained RT-PCR amplification of the SPO_PG027-SPO_PG09 region. Despite this, no amplification could be obtained between this operon and its downstream rplO or upstream $r p m D$ genes. The latter result was expected because of a putative Rho-independent transcriptional terminator identified by the TransTerm software (Figure 2, Panel B).

The neighbouring genes in the operon structures of the newly annotated CDSs may infer plausible hints about the functions of the novel detected genes. This is the case for SPO_PG026, which we have shown here to be co-transcribed with the SPO_3673 gene. We have previously shown that the latter encodes an RTX-like toxin which is abundantly secreted in the culture medium [24]. RTX operons always comprise the toxin gene itself together with other genes involved in toxin activation and export [25]. SPO_PG026 could be involved in such activation or export functions. SPO_PG018 is also part of a putative RTX-like operon. We detected, by RT-PCR amplification, an operon structure comprising this gene and its flanking SPO_0490 and SPO_0491 genes. SPO_0490 shows sequence similarities with calcium-binding RTX toxins. This protein has a putative signal peptide for export as identified by the SignalP predictor software [26].

RT-PCR amplification was also performed for the SPO_PG023 and SPO_1339 couple, possibly with a linked function. SPO_1339, a signal-recognition, particle-docking protein, is located just upstream of SPO_PG023. Another operon structure detected was that of SPO_PG010 with the upstream SPO_2211SPO_2212-SPO_2213 genes. These three genes encode the determinants of branched-chain amino acid catabolism (acyl-CoA dehydrogenase, enoyl-CoA hydratase and 3-hydroxyisobutyrate dehydrogenase). SPO_PG010 shows similarities with only a few proteins found in other Roseobacter members (Oceanicola batsensis HTCC2597, Roseovarius sp. HTCC2601 and Sagittula stellata E-37). The loci encoding these far-homologues 
are also located close to genes with functions related to branched-chain amino acid metabolism. However, the genomic context is not always similar to that found in $R$. pomeroyi. On the other hand, we found that SPO_PG022 is co-transcribed with its downstream gene, SPO1095, and upstream gene, SPO1094, a putative propionyl-CoA carboxylase. SPO1094 converts propionylCoA (a derivative product from branched-chain amino acid catabolism) to methylmalonyl-CoA (a precursor of the citric acid cycle component, succinyl-CoA). Strikingly, SPO_PG022 shares similarity with one other annotated gene, in the Roseobacter sp MED193, which also shows the same putative operon structure.

SPO_PG005 and SPO_PG006, shown in Figure 4, and SPO_PG016, are encoded in the close neighbourhood of the operon structures encoding essential enzymes of the central metabolic citric acid cycle in $R$. pomeroyi. Interestingly, SPO_PG006 and SPO_PG016 were shown to be co-transcribed with the genes encoding these enzymes. SPO_PG005, which is co-transcribed with the SPO_0345 gene (whose product has no assigned function), is independently transcribed from its flanking operons, sucCD and sucAB (Figure 4). SPO_PG006 belongs to the $s u c C D$ operon and is located between the sucC and sucD genes. Figure 4 shows the genomic context for the sucCDAB genes for seven other Rhodobacteraceae bacteria. The presence of additional CDSs in the sucCD operon is exclusive to the Roseobacter strains. Conversely, the minimal structure observed in other Rhodobacteraceae is the common rule amongst bacteria belonging to other clades (e.g. Pseudomonas or Bacillus). In all Roseobacter species that were compared, we observed a variable pattern of novel genes inserted within the $\operatorname{suc} C D$ operon and in the intergenic region between $s u c A B$ and $s u c C D$. These novel genes are poorly conserved between the different Roseobacter members, as shown in Figure 4. The gene encoding SPO_PG016 is found inserted within the succinate dehydrogenase operon, $s d h C D A B$. We demonstrated its cotranscription with its flanking genes, SPO0360 and SPO0361, by a specific RT-PCR amplification. The presence of non-conserved genes included in the $s d h C D A B$ operon is also common amongst Roseobacter members, but not in other bacteria.

\section{Discussion and conclusions}

From the large dataset of MS/MS spectra (1 117 372) recorded for proteome samples of $R$. pomeroyi cultivated in various conditions, we identified thirty-nine newly annotated genes and nine wrongly described ORFs. We also corrected seventy-four start codons and described five sequencing errors (a base insertion in all cases) that consequently modified the characteristics of the genes encoded at these loci. Because of its environmental relevance, the Roseobacter clade is currently subject to intense sequencing efforts [27-31]. However, because of the large diversity of this bacterial group, there are insufficient numbers of near-related genome sequences to improve their annotations by comparative genomics alone. Here, we show the importance of proteogenomics input for a better characterization of bacterioplankton.

We noted that the number of annotation inaccuracies, in terms of structural annotation, is rather large for $R$. pomeroyi genome annotation. This is similar to previous proteogenomic reports for Shewanella or Mycobacterium bacteria that resulted in thirty-eight and twentynine new annotations, respectively $[19,32]$. In contrast, a recent proteogenomic study carried out on the enterobacterium Yersinia pestis identified only four novel genes [20]. As enterobacteria are the most extensively studied organisms, and numerous genomes from the Enterobacteriaceae family have now been sequenced and annotated, it is reasonable to consider that their genomes are amongst the best for accuracy and reliability. This is in full agreement with the proteogenomic data presented by Payne et al. [20]. Here we have shown that even highly expressed genes and operons with potentially important cellular roles were missed during the genome annotation of $R$. pomeroyi. The majority of annotation problems come from the identification of CDSs exclusive to a small number of organisms, as comparative genomics is not useful in such a case in confirming the ORF prediction. Their validation requires additional experimental evidence, such as described here. Blending data from complementary approaches, such as protein characterization by tandem mass spectrometry and transcriptomic evidence, is time consuming but results in stronger evidence for small genes. In terms of mass spectrometry, 'one-hit-wonders' are proteins identified with only one, non-redundant peptide tag. They are usually proteins with low molecular weight that are able to generate only a few tryptic peptides. Depending on the score of MS/MS spectrum assignment, these hits may be difficult to ascertain confidently and require manual validation. Gupta et al [33] proposed a method to validate one-hit-wonders using comparative proteogenomics, but this requires the recording of various MS/MS datasets on several species. Here, we used RT-PCR to detect the expression of several CDSs identified with only one peptide. In this way we obtained evidence that the locus was being expressed, giving higher confidence to the assignment. This method proved to be effective, with the addition of five novel genes to our list.

Another frequent problem encountered during genome annotation is the identification of a CDS located in two different reading frames that clearly encodes a unique, conserved protein. This can be either a real 
frameshift process occurring for the regulation of protein synthesis, an artefact resulting from a sequencing error, or a pseudogene that has been recently inactivated. As we identified in the present study peptides in different reading frames at the same loci (e.g. SPO_PG036 and SPO_PG037), we confirmed the production of the polypeptides encoded and discounted the existence of pseudogenes. We checked systematically the sequences of the five loci and found in all cases that the plausible frameshifts resulted from sequencing errors. The insertion of an extra nucleotide in the sequence produced a slippage of the coding region to another reading frame in the five cases. This was expected as frameshifts are rare processes of regulation and usually down-regulate the protein synthesis in bacteria, while they are frequent in Archaea or viruses $[34,35]$. The number of sequencing errors found in the $R$. pomeroyi genome sequence also supports the current idea of re-sequencing genomes which were established a decade ago [14]. Here we have confirmed the input of proteogenomics to indicate the specific loci that need such sequence re-evaluation which has already been highlighted by others [36].

It is worth noting that ortho-proteogenomic extension of the corrected annotations to phylogenetically-related microorganisms reinforces the interest of proteogenomic studies for poorly studied bacterial phyla. Ortho-proteogenomic analyses have, to date, been limited to only two genera, Mycobacterium [19] and Yersinia [20], and have not been extended beyond this taxonomic level. In the present work, we exploited the MS/MS data combined with comparative genomics to extend re-annotations for genomes from higher taxonomic ranks. Although all sequenced members of the Roseobacter clade are distantly related, they all form a robust cluster with a high rate of similarities at the 16S RNA nucleotide sequence level [37]. We have successfully extended the identified $\mathrm{N}$-terminal annotation of the 486 proteins detected in $R$. pomeroyi to 9887 homologous genes in the thirty-six sequenced Roseobacter isolates, corresponding to nineteen distinct genera. In this way, 1082 genes that were wrongly annotated were confidently corrected. This represents $11 \%$ of the total number of ORFs considered. To highlight the importance of manual curation of genome annotations, the rate of erroneous $\mathrm{N}$-terminal identifications decreased to $6.8 \%$ when considering only the four complete Roseobacter genomes included in this study. These error rates are probably slightly underestimated as we only considered the conserved and obvious corrections. It is important to note that the full rate of badly annotated $\mathrm{N}$-terminal genes established on the well-annotated genome of $R$. pomeroyi was $12.8 \%$. A more comprehensive annotation of the clade could only be accomplished by integrating a comparative proteogenomic analysis of various Roseobacter strains, as previously carried out with the genus Shewanella (Gupta et al 2008).

Finally, the identification of operon structures by RTPCR has given insights of the plausible function of the novel proteins identified in the present study. Bacterial genomes are usually well structured and regulated in the form of operons. Remarkably, we found that most of the novel, proteogenomic-detected CDSs were identified in operons encoding catabolic functions for amino acid degradation, RTX-like secreted proteins or central citric cycle metabolism. Because most of the biological conditions were carried out in a peptide broth [18], this catabolism is privileged and such discovery may be advantaged. Whether the genes encoded in the close neighbourhood of genes specifying RTX-like toxins are part of the protein secretion system or associated factors is an interesting question, as such toxins can be abundantly secreted, as previously shown [24,38]. Moreover, we identified novel CDSs with no ascribable function in operons encoding essential determinants of the citric acid cycle. These novel CDSs are not at all conserved among other Roseobacter members, but their presence is a common topic restricted to members of this clade. Whether these genes encode proteins that enhance this central metabolic cycle in these bacteria or are opportunistic genes that specifically appear in this operon because of the advantage of their high expression is an open question. The presence of the novel proteins found in the citric acid cycle operon in $R$. pomeroyi could represent snapshots of how novel proteins with novel specific functions arise during evolution.

Systematic listing of CDSs in numerous microorganisms, with the help of proteogenomic evidence, should increase the accuracy of annotation software. As demonstrated here, proteogenomic evidences from bacteria belonging to orders that have, thus far, been poorly characterized, such as the Roseobacter clade, are necessary to improve genome and even metagenome annotations. Ortho-proteogenomic annotation extension to a whole bacterial clade has proven here to be highly valuable. Such extension could also be applied to metagenome data, taking into account higher constraints.

\section{Methods \\ MS/MS data compilation}

An extensive proteomic analysis was applied to $R$. pomeroyi consisting in 136 nanoLC-MS/MS runs acquired on proteomes and subproteomes from cells grown under 30 different culture conditions $[18,24]$. Briefly, we tried out exponential and stationary culture phases in rich and poor media, with different incubation parameters (salt concentration, temperature, microaerobiosis, pelleted cells, plate growth), and stresses (UV 
illumination, the presence of aromatic compounds). Cellular subproteomes were also included (cellular membrane, phosphocellulose enriched fraction and exoproteome). In addition, a novel set of 75 nanoLCMS/MS runs were acquired from samples prepared from five cellular extracts of cells grown in exponential phase in marine broth and then subjected to UV stress, concentration $(100 \times$ concentrated cells), or grown on benzoate or in presence of naphthalene, and then resolved by SDS-PAGE. Cell cultures, protein extraction, SDS-PAGE and trypsin digestion were performed as previously described [18,39]. MS/MS analysis was carried out with a LTQ-Orbitrap XL hybrid mass spectrometer (ThermoFisher) coupled to an UltiMate 3000 LC system (Dionex-LC Packings) using the same parameters as already published [23].

\section{MS/MS database search}

A total of 1,117,372 MS/MS spectra were compiled from the 211 nanoLC-MS/MS runs. These were first tentatively assigned using an ORF database containing all the sixframe translated stop-to-stop protein sequences coded in the 4.6-kb genome of $R$. pomeroyi (NC_003911 and NC_006569, [22]). This ORF database contained 61,206 protein sequences of over 40 amino acids in size, as well as the bovine trypsin sequence and 22 of the most common human keratin contaminants. The total sequences presented 7,298,532 amino acid residues. This large database contains a large percentage of non-real protein sequences. Peak lists were generated with the MASCOT DAEMON software (version 2.2.2) from Matrix Science using the extract_msn.exe data import filter from the Xcalibur FT package (version 2.0.7) from ThermoFisher. Data import filter options were set at: 400 (minimum mass), 5000 (maximum mass), 0 (grouping tolerance), 0 (intermediate scans), and 1000 (threshold). MS/MS assignments were performed using the MASCOT search engine (version 2.3.2, Matrix Science) against the local database. Searches for tryptic peptides were performed with the following parameters: a mass tolerance of $5 \mathrm{ppm}$ on the parent ion and $0.5 \mathrm{Da}$ on the MS/MS, static modifications of carbamidomethylated Cys (+57.0215), and dynamic modification of oxidized Met $(+15.9949)$. The maximum number of missed cleavage for trypsin was set at 1 . All peptide matches with a score above its peptidic identity threshold set at $p<0.005$ and rank 1 were filtered by the IRMa 1.26.1 software [40]. A total of 22,040 non-redundant peptides were found belonging to 2,550 polypeptide sequences. A more restricted database consisting of i) the 4,252 stop-to-stop ORFs corresponding to the annotated CDS [22] and ii) the additional sequences pointed by the first MASCOT search (173) was constructed. Thus, this database comprises 4,425 stop-to-stop ORFs sequences, totaling 1,584,061 amino acid residues. The whole MS/MS spectra dataset was searched against this reduced database using the same parameters. A total of 594,902 spectra were confidently assigned evidencing 22,805 non-redundant peptides (Additional file 1). Considering that a protein was validated when at least two different confident peptides were detected, 2,006 protein sequences were listed (Additional file 2). A total false positive rate of $0.8 \%$ and $0.2 \%$ for identification of peptide and polypeptide, respectively, was estimated using the reverse decoy database. In order to catalogue the most $\mathrm{N}$-terminal peptides of the proteins, we performed a new MASCOT search on the reduced database for listing semi-tryptic peptides. We also searched for i) modification of translation starts that could arise because of an insertion of a methionine residue at GTG and TTG translation initiation codons, and ii) protein maturation consisting in the processing of the initiator Methionine, as described previously [23]. The resulting semi-tryptic peptides were further filtered to keep only those corresponding plausible most $\mathrm{N}$-terminal peptides.

\section{Nucleic acid manipulation}

Genomic DNA extraction of $R$. pomeroyi cells was performed using the DNeasy Blood and Tissue Kit (Qiagen) following manufacturer instructions for gram-negative bacteria. DNA PCR amplification was carried out with standard conditions using Taq DNA polymerase (Roche). All primers used for specific PCR amplification of the different targets are listed in Supplementary Additional file 3. Sequencing procedure was done on specific amplified sequence purified with the QIAquick PCR purification kit (Qiagen) and was performed systematically on at least two independent amplification products. Reaction was carried out using Dye Terminator Cycle Sequencing Quick Start kit (Beckman Coulter) and analysed on a CEQ 2000 XL DNA analysis system, as previously described [41]. RNA protect Bacteria Reagent (Qiagen) was used during cell harvesting in order to stabilize the RNA and avoid any degradation. RNA extraction was performed with the RNeasy mini kit (Qiagen) using lysozyme for bacterial lysis and RNase-free DNase (Qiagen) for DNA digestion. PCR amplification was carried out systematically to test for total DNA digestion prior to RT-PCR reaction. RNA quantification was performed with a NanoDrop ND-1000 spectrophotometer. RT-PCR was carried out using the Titan One Tube RTPCR kit (Roche). PCR amplifications were visualised on $2 \%$ agarose gels stained with ethidium bromide.

\section{Genome and protein sequence analysis}

Visualization of genome sequences and gene positioning was done with the Artemis browser (release 12.0,[42]). The TransTerm software [43] was used to search for Rhoindependent signals for transcription termination. Local 
BLAST analysis was carried out using the BioEdit sequence editor v7.0.9.0 [44]. We carried out a local TBLASTN analysis searching amongst the other 36 sequenced Roseobacters for genes specifying similar proteins to those detected by proteogenomics in $R$. pomeroyi. These strains were: Citriecella sp. SE45, Dinoroseobacter shibae DFL 12, Jannaschia sp. CCS1, Loktanella vestfoldensis SKA53, Maritimibacter alkaliphilus HTCC2654, Oceanibulbus indolifex HEL-45, Oceanicola batsensis HTCC2597, Oceanicola granulosus HTCC2516, Octadecabacter antarcticus 238, Octadecabacter antarcticus 307, Pelagibaca bermudensis HTCC2601, Phaeobacter gallaeciensis 2.10, Phaeobacter gallaeciensis BS107, Phaeobacter sp. Y4I, Rhodobacteraceae KLH11, Rhodobacterales bacterium HTCC2150, Rhodobacterales bacterium HTCC2255, Rhodobacterales HTCC2083, Roseobacter denitrificans OCh 114, Roseobacter litoralis Och 149, Roseobacter sp. AzwK-3b, Roseobacter sp. CCS2, Roseobacter sp. GAI101, Roseobacter sp. MED193, Roseobacter sp. SK209-2-6, Roseovarius nubinhibens ISM, Roseovarius sp. 217, Roseovarius sp. TM1035, Ruegeria lacuscaerulensis ITI-1157, Ruegeria sp. R11, Ruegeria sp. TM1040, RuegeriaTrich CH4B, Sagittula stellata E-37, Sulfitobacter sp. EE-36, Sulfitobacter sp. NAS-14.1 and Thalassiobium R2A62. The E-value cutoff was set at $<10^{-20}$. We also searched protein homologues in the other 36 Roseobacter proteomes with a local BLASTP tool $\left(\mathrm{E}\right.$-value $\left.<10^{-30}\right)$ in order to check specifically their most probable $\mathrm{N}$-termini.

\section{Nucleotide and protein sequences}

The novel Ruegeria pomeroyi DSS-3 nucleotide sequences reported in this paper have been deposited in the EMBL/GenBank Nucleotide Sequence Database under the accession numbers FR852579 (SPO_PG036) FR852580 (SPO_PG037), FR852581 (SPO_PG038), FR852582 (SPO_PG039), and FR852583 (SPO1905).

\section{Additional material}

Additional file 1: Non-redundant peptide list detected by MS/MS spectral attributions $(\mathbf{p}<0.005)$. The excel table presents the characteristics of all the non-redundant peptides detected (22805 items) and their corresponding proteins.

Additional file 2: Stop-to-stop polypeptides detected by MS/MS. The excel table presents the whole list (2547 items) of ORFs detected by MS/ MS with their annotated function and sequence, as well as the number of non-redundant peptides assigned to each of these ORFs.

Additional file 4: List of novel genes encoded in the genome of $R$. pomeroyi detected by proteogenomics in this study. The excel table presents the 39 new genes found in the present study and their characteristics. The corresponding ORF, the number of non-redundant peptides, the number of MS/MS spectra assigned, the plausible protein sequence, and the conditions where each product has been detected are listed for the 39 genes.

Additional file 5: Annotated CDS demonstrated to be longer due to the detection of tryptic peptides situated further $\mathrm{N}$-ter than previously annotated. The excel table presents the evidences for 10 annotated CDS that should be revised in terms of $\mathrm{N}$-terminus. The corresponding ORF label, the number of non-redundant peptides, the new proteomic-corrected CDS sequence and the corresponding peptide evidences (peptide query, peptide sequence, peptide score) are indicated for each of these $10 \mathrm{CDS}$.

Additional file 6: Semi-tryptic peptides representing the most N-ter sequence of the stop-to-stop CDS. The excel table presents the characteristics of all the semi-tryptic peptides detected which correspond to the most N-terminal sequence of the stop-to-stop ORF (22805 items) and their corresponding proteins. We distinguished the peptides starting with a methionine residue encoded by an ATG start codon (lines 5-136), those starting from the residue just after a removed methionine encoded by an ATG start codon (lines 138-463), the peptides starting with a methionine residue encoded by an GTG start codon (lines 465471), those starting from the residue just after a removed methionine encoded by an GTG start codon (lines 473-489), and those starting from the residue just after a removed methionine encoded by an $\Pi \mathrm{TG}$ start codon (lines 491-494).

Additional file 7: Ortho-proteogenomic based analysis extending the $\mathrm{N}$-ter corrections done on $R$. pomeroyi to 36 other isolates of the Roseobacter clade. The excel table presents the extension to 36 isolates for each of R. pomeroyi re-annotated genes (9887 proposals). The e-value obtained by BLAST is indicated for each homologue, as well as its $\mathrm{N}$-terminus (60 first residues), the new CDS length, and its location on the corresponding genome.

Additional file 3: Primers used throughout the study for PCR amplifications. The excel table presents the 37 primers used in the study for PCR amplification of specific genomic loci and their characteristics (sequence, position, names, melting temperature, targets).

\section{Abbreviations}

ORFs: Open Reading Frames; CDS: coding domain sequences; MS/MS: tandem mass spectrometry.

\section{Acknowledgements}

JA Christie-Oleza was supported by a fellowship from the Fundación Ramón Areces. We thank the Commissariat à l'Energie Atomique et aux Energies Alternatives, the Fundación Ramón Areces, and the Ministère des Affaires Etrangères et Européennes, République Française (Partenariat Hubert Curien, Picasso Program) for financial support. We also thank Vanessa Gardes for help during experimentation in the course of her Master1 training period.

\section{Authors' contributions}

JC-O designed and performed the experiments of microbiology and proteomics, and analyzed all the data. GC contributed to mRNA analysis and sequence verification. JA conceived, coordinated the study and participated in the analysis of the proteomic data. JC-O and JA wrote the manuscript. All authors read and approved the final manuscript.

Received: 25 July 2011 Accepted: 15 February 2012 Published: 15 February 2012

\section{References}

1. Fleischmann RD, Adams MD, White O, Clayton RA, Kirkness EF, Kerlavage AR, Bult CJ, Tomb JF, Dougherty BA, Merrick JM, et al: Wholegenome random sequencing and assembly of Haemophilus influenzae Rd. Science 1995, 269(5223):496-512.

2. Kyrpides NC: Fifteen years of microbial genomics: meeting the challenges and fulfilling the dream. Nat Biotech 2009, 27(7):627-632.

3. Armengaud J: A perfect genome annotation is within reach with the proteomics and genomics alliance. Curr Opin Microbiol 2009, 12:292-300.

4. Poptsova MS, Gogarten JP: Using comparative genome analysis to identify problems in annotated microbial genomes. Microbiol 2010, 156(7):1909-1917.

5. Nielsen P, Krogh A: Large-scale prokaryotic gene prediction and comparison to genome annotation. Bioinformatics 2005, 21(24):4322-4329. 
6. Armengaud J, Bland C, Christie-Oleza JA, Miotello G: Microbial proteogenomics, gaining ground with the avalanche of genome sequences. J Bacteriol Parasitol 2011, S3-001.

7. Armengaud J: Proteogenomics and systems biology: quest for the ultimate missing parts. Expert Rev Proteomics 2010, 7:65-77.

8. Mou X, Sun S, Edwards RA, Hodson RE, Moran MA: Bacterial carbon processing by generalist species in the coastal ocean. Nature 2008, 451(7179):708-711.

9. Denoeud F, Aury JM, Da Silva C, Noel B, Rogier O, Delledonne M, Morgante M, Valle G, Wincker P, Scarpelli C, et al: Annotating genomes with massive-scale RNA sequencing. Genome Biol 2008, 9:R175.

10. Ahrens CH, Brunner E, Qeli E, Basler K, Aebersold R: Generating and navigating proteome maps using mass spectrometry. Nat Rev Mol Cell Biol 2010, 11:789-801.

11. Gstaiger M, Aebersold R: Applying mass spectrometry-based proteomics to genetics, genomics and network biology. Nat Rev Genet 2009, 10(9):617-627.

12. Yates JR, Eng JK, McCormack AL: Mining genomes: correlating tandem mass spectra of modified and unmodified peptides to sequences in nucleotide databases. Anal Chem 1995, 67(18):3202-3210.

13. Jaffe JD, Stange-Thomann N, Smith C, DeCaprio D, Fisher S, Butler J, Calvo S, Elkins T, FitzGerald MG, Hafez N, et al: The complete genome and proteome of Mycoplasma mobile. Genome Res 2004, 14(8):1447-1461.

14. de Groot $A$, Dulermo R, Ortet $P$, Blanchard $L$, Guerin $P$, Fernandez $B$, Vacherie $B$, Dossat $C$, Jolivet $E$, Siguier $P$, et al: Alliance of proteomics and genomics to unravel the specificities of Sahara bacterium Deinococcus deserti. PLoS Genet 2009, 5(3):e1000434.

15. Zivanovic Y, Armengaud J, Lagorce A, Leplat C, Guerin P, Dutertre M, Anthouard V, Forterre P, Wincker P, Confalonieri F: Genome analysis and genome-wide proteomics of Thermococcus gammatolerans, the most radioresistant organism known amongst the Archaea. Genome Biol 2009, 10(6):R70.

16. Borchert N, Dieterich C, Krug K, Schütz W, Jung S, Nordheim A, Sommer RJ, Macek B: Proteogenomics of Pristionchus pacificus reveals distinct proteome structure of nematode models. Genome Res 2010, 20(6):837-846.

17. Qiu Y, Cho B-K, Park YS, Lovley D, Palsson BA, Zengler K: Structural and operational complexity of the Geobacter sulfurreducens genome. Genome Res 2010, 20(9):1304-1311.

18. Christie-Oleza JA, Fernandez B, Nogales B, Bosch R, Armengaud J: Proteomic insights into the lifestyle of an environmentally relevant marine bacterium. ISME J 2012, 6(1):124-135.

19. Gallien Sb, Perrodou E, Carapito C, Deshayes C, Reyrat J-M, Van Dorsselaer A, Poch O, Schaeffer C, Lecompte O: Ortho-proteogenomics: Multiple proteomes investigation through orthology and a new MSbased protocol. Genome Res 2009, 19(1):128-135.

20. Payne S, Huang S-T, Pieper R: A proteogenomic update to Yersinia: enhancing genome annotation. BMC Genomics 2010, 11(1):460.

21. Buchan A, Gonzalez JM, Moran MA: Overview of the marine Roseobacter lineage. Appl Environ Microbiol 2005, 71(10):5665-5677.

22. Moran MA, Buchan A, Gonzalez JM, Heidelberg JF, Whitman WB, Kiene RP, Henriksen JR, King GM, Belas R, Fuqua C, et al: Genome sequence of Silicibacter pomeroyi reveals adaptations to the marine environment. Nature 2004, 432(7019):910.

23. Baudet M, Ortet P, Gaillard J-C, Fernandez B, Guerin P, Enjalbal C, Subra G, de Groot A, Barakat M, Dedieu A, et al: Proteomics-based refinement of Deinococcus deserti genome annotation reveals an unwonted use of non-canonical translation initiation codons. Mol Cell Proteomics 2010, 9(2):415-426.

24. Christie-Oleza JA, Armengaud J: In-depth analysis of exoproteomes from marine bacteria by shotgun liquid chromatography-tandem mass spectrometry: the Ruegeria pomeroyi DSS-3 case-study. Mar Drugs 2010, 8:2223-2239

25. Linhartová I, Bumba L, Mašín J, Basler M, Osička R, Kamanová J, Procházková K, Adkins I, Hejnová-Holubová J, Sadiliková L, et al: RTX proteins: a highly diverse family secreted by a common mechanism. FEMS Microbiol Rev 2010, 34:1076-1112.

26. Bendtsen JD, Nielsen H, von Heijne G, Brunak S: Improved prediction of signal peptides: SignalP 3.0. J Mol Biol 2004, 340(4):783-795.
27. Kang I, Oh HM, Vergin KL, Giovannoni SJ, Cho JC: Genome sequence of the marine alphaproteobacterium HTCC2150, assigned to the Roseobacter clade. J Bacteriol 2010, 192(23):6315-6316.

28. Kang I, Vergin KL, Oh HM, Choi A, Giovannoni SJ, Cho JC: Genome sequence of strain HTCC2083, a novel member of the marine clade Roseobacter. J Bacteriol 2011, 193(1):319-320.

29. Thrash JC, Cho JC, Ferriera S, Johnson J, Vergin KL, Giovannoni SJ: Genome sequences of Pelagibaca bermudensis HTCC2601T and Maritimibacter alkaliphilus HTCC2654T, the type strains of two marine Roseobacter genera. J Bacteriol 2010, 192(20):5552-5553.

30. Thrash JC, Cho JC, Vergin KL, Giovannoni SJ: Genome sequences of Oceanicola granulosus HTCC2516(T) and Oceanicola batsensis HTCC2597 (TDelta). J Bacteriol 2010, 192(13):3549-3550

31. Wagner-Dobler I, Ballhausen B, Berger M, Brinkhoff T, Buchholz I, Bunk B, Cypionka H, Daniel R, Drepper T, Gerdts G, et al: The complete genome sequence of the algal symbiont Dinoroseobacter shibae: a hitchhiker's guide to life in the sea. ISME J 2010, 4(1):61-77.

32. Gupta N, Tanner S, Jaitly N, Adkins JN, Lipton M, Edwards R, Romine M, Osterman A, Bafna V, Smith RD, et al: Whole proteome analysis of posttranslational modifications: applications of mass-spectrometry for proteogenomic annotation. Genome Res 2007, 17(9):1362-1377.

33. Gupta N, Benhamida J, Bhargava V, Goodman D, Kain E, Kerman I, Nguyen N, Ollikainen N, Rodriguez J, Wang J, et al: Comparative proteogenomics: Combining mass spectrometry and comparative genomics to analyze multiple genomes. Genome Res 2008, 18(7):1133-1142.

34. Baranov PV, Fayet O, Hendrix RW, Atkins JF: Recoding in bacteriophages and bacterial IS elements. Trends Genet 2006, 22(3):174-181.

35. Cobucci-Ponzano B, Guzzini L, Benelli D, Londei P, Perrodou E, Lecompte O, Tran D, Sun J, Wei J, Mathur EJ, et al: Functional characterization and high-throughput proteomic analysis of interrupted genes in the archaeon Sulfolobus solfataricus. J Proteome Res 2010, 9(5):2496-2507.

36. Deshayes C, Perrodou E, Gallien S, Euphrasie D, Schaeffer C, VanDorsselaer A, Poch O, Lecompte O, Reyrat JM: Interrupted coding sequences in Mycobacterium smegmatis: authentic mutations or sequencing errors? Genome Biol 2007, 8(2):R20.

37. Newton RJ, Griffin LE, Bowles KM, Meile C, Gifford S, Givens CE, Howard EC, King $E$, Oakley $C A$, Reisch $C R$, et al: Genome characteristics of a generalist marine bacterial lineage. ISME J 2010, 4(6):784-798.

38. Christie-Oleza JA, Pina-Villalonga JM, Bosch R, Nogales B, Armengaud J: Comparative proteogenomics of twelve Roseobacter exoproteomes reveals different adaptive strategies amongst these marine bacteria. Mol Cell Proteomics 2012, 11(2):M111.013110.

39. Clair G, Roussi S, Armengaud J, Duport C: Expanding the known repertoire of virulence factors produced by Bacillus cereus through early secretome profiling in three redox conditions. Mol Cell Proteomics 2010, 9(7):1486-1498.

40. Dupierris V, Masselon C, Court M, Kieffer-Jaquinod S, Bruley C: A toolbox for validation of mass spectrometry peptides identification and generation of database: IRMa. Bioinformatics 2009, 25(15):1980-1981.

41. Armengaud J, Fernandez B, Chaumont Vr, Rollin-Genetet Fo, Finet Sp, Marchetti C, Myllykallio H, Vidaud C, Pellequer J-L, Gribaldo S, et al: Identification, purification, and characterization of an eukaryotic-like phosphopantetheine adenylyltransferase in the hyperthermophilic archaeon Pyrococcus abyssi. J Biol Chem 2003, 278(33):31078-31087.

42. Rutherford K, Parkhill J, Crook J, Horsnell T, Rice P, Rajandream M-Al, Barrell B: Artemis: sequence visualization and annotation. Bioinformatics 2000, 16(10):944-945.

43. Jacobs GH, Chen A, Stevens SG, Stockwell PA, Black MA, Tate WP, Brown CM: Transterm: a database to aid the analysis of regulatory sequences in mRNAs. Nucleic Acids Res 2009, 37:D72-D76.

44. Hall TA: BioEdit: a user-friendly biological sequence alignment editor and analysis program for Windows 95/98/NT. Nucl Acids Symp Ser 1999, 41:95-98.

doi:10.1186/1471-2164-13-73

Cite this article as: Christie-Oleza et al:: High-throughput proteogenomics of Ruegeria pomeroyi: seeding a better genomic annotation for the whole marine Roseobacter clade. BMC Genomics 2012 13:73. 